\title{
Conformational relaxation of single polymer chains confined in a two-dimensional plane studied by scanning near-field optical microscopy
}

\author{
Hiroyuki Aoki ${ }^{1,2}$, Ryojun Sekine ${ }^{1}$, Tatsuya Iwamoto $^{1}$ and Shinzaburo Ito ${ }^{1,2}$
}

The conformational rearrangement of poly(methyl methacrylate) (PMMA) chains confined in a two-dimensional monolayer to a three-dimensional random coil was studied by the shape and segment density analysis of an individual PMMA chain, and was directly observed by scanning near-field optical microscopy. A perylene-labeled PMMA chain was dispersed in the unlabeled PMMA monolayer, after which it was placed on a thick film of unlabeled PMMA and annealed above the glass transition temperature. Although the polymer chain of the monolayer penetrated into the bulk substrate with thermal activation, the radius of gyration in the lateral direction was maintained throughout the annealing process. The diffusion rate in the depth direction was much faster than that of the translational diffusion of the entire polymer chains, suggesting that an unstable twodimensional conformation of the polymer chain relaxed into a three-dimensional one. This indicates that the polymer chains confined in a monolayer interlaced with the surrounding chains and expanded mainly toward the depth direction in the relaxation process, while maintaining the dimensions of the averaged radius of gyration in the lateral direction.

Polymer Journal (2010) 42, 124-130; doi:10.1038/pj.2009.323; published online 23 December 2009

Keywords: chain conformation; Langmuir-Blodgett film; relaxation; scanning near-field optical microscopy; single polymer chain

\section{INTRODUCTION}

Polymer monolayers have attracted much attention because they allow one to fabricate well-defined nanometric architectures with thermal durability compared with those made from conventional long-chain fatty acids. ${ }^{1}$ Poly(alkyl methacrylate) is well-known to form a stable monolayer at the air-water interface because it has an amphiphilic ester group in each monomer unit. ${ }^{2}$ The main chain is constrained on the water surface, resulting in a restricted conformation in quasi-two dimensions. Thermal stability of the polymer monolayer is of crucial importance from the viewpoint of the practical application of such highly controlled molecular assemblies. However, the two-dimensional conformation of polymer chains in a monolayer is entropically unstable compared with three-dimensional conformation. Therefore, they suffer inevitably from thermal relaxation after deposition on solid substrates. In the last few decades, the thermal stability of Langmuir-Blodgett films has been examined by polarized infrared spectroscopy, ${ }^{3,4}$ the optical second-harmonic generation method, ${ }^{5}$ small-angle X-ray scattering, ${ }^{6,7}$ $\mathrm{X}$-ray reflectivity ${ }^{8,9}$ and the energy transfer technique. ${ }^{10,11}$ Several research groups reported the structural relaxation of polymer LangmuirBlodgett films in nanometer dimensions by thermal treatment and suggested that chain conformation was rearranged from a twodimensionally restricted form to a three-dimensional equilibrium conformation by the local motion of polymer segments. ${ }^{8-10}$
In contrast to the methods used in previous studies, the direct observation of individual chains is expected to provide clear evidence on chain conformation and would be helpful in understanding the previous results obtained from ensemble-averaged measurements. Recently, several studies on the direct observation of a single polymer chain using atomic force microscopy have been reported. The high spatial resolution of atomic force microscopy enables us to observe the contour of a single polymer chain. ${ }^{12-14}$ As atomic force microscopy detects only the topographic information of the sample surface, it is unable to observe the single polymer chain embedded in a bulk medium. To observe single chain conformation, we must use a novel technique to distinguish the objective polymer chain from the surrounding ones. Fluorescence labeling is a promising method for overcoming this requirement. ${ }^{15,16}$ The introduction of a small amount of fluorescence chromophores into a polymer chain enables us to image single fluorescent chains owing to the high sensitivity of fluorescence detection. However, the spatial resolution of a conventional fluorescence microscope is limited to half of the excitation wavelength by the diffraction barrier. ${ }^{17}$ This has limited its application in single chain imaging, except for huge bio-macromolecules such as DNA. ${ }^{15,16}$ Scanning near-field optical microscopy (SNOM) has been developed as a novel technique for achieving a high spatial resolution beyond the diffraction limit of light. ${ }^{18-20}$ SNOM is a method of

${ }^{1}$ Department of Polymer Chemistry, Kyoto University, Nishikyo, Kyoto, Japan and ${ }^{2}$ Advanced Biomedical Engineering Research Unit, Kyoto University, Nishikyo, Kyoto, Japan Correspondence: Professor H Aoki, Department of Polymer Chemistry, Kyoto University, Kyoto-Daigaku-Katsura, Nishikyo, Kyoto 615-8510, Japan. E-mail: aoki@photo.polym.kyoto-u.ac.jp 
scanning probe microscopy, which is equipped with the probe tip having an aperture smaller than the wavelength of light. The near-field light emanating from such a small aperture is confined to the vicinity of the probe tip end, which allows us to illuminate the nanometric space under the sample surface. Therefore, SNOM is suitable for investigating polymer chain conformations in a bulk medium. ${ }^{21-26}$

In this study, we present the direct measurement of the real-space image of an individual poly(methyl methacrylate) (PMMA) chain in a monolayer using SNOM. PMMA forms a monolayer with a thickness of ca. $1 \mathrm{~nm}$ on water, as each monomer unit is restricted on the water surface. ${ }^{13,27,28}$ The monolayer of PMMA deposited on a substrate by the Langmuir-Blodgett method can be regarded as a model of the polymer chain confined in two dimensions. We discuss the conformational relaxation of PMMA chains in a monolayer by thermal treatment on the basis of the annealing time dependence of the chain dimension and segment distribution. SNOM observation in the depth direction is also carried out and the thermally induced rearrangement of the segment distribution is investigated in comparison with the translational diffusion of the whole chain.

\section{EXPERIMENTAL PROCEDURE}

\section{Sample preparation}

Perylene-labeled poly(methyl methacrylate) (PMMA-Pe) was synthesized by radical copolymerization of methyl methacrylate (Wako Pure Chemical Industries, Osaka, Japan) and 3-perylenyl methyl methacrylate. ${ }^{29}$ Details of the synthesis are described elsewhere. ${ }^{23,24}$ The weight average molecular weight, $M_{\mathrm{w}}$, and the molecular weight distribution, $M_{\mathrm{w}} / M_{\mathrm{n}}$, were $3.0 \times 10^{6}$ and 1.3 , respectively. The molar fraction of the perylene moiety introduced in a PMMA chain was evaluated to be $0.84 \%$ from ultraviolet-visible absorption measurement. PMMA-Pe was dispersed in a monolayer of unlabeled PMMA $\left(M_{\mathrm{w}}=2.2 \times 10^{6}\right.$, American Polymer Standards) in concentrations from 0.1 to $0.5 \mathrm{wt} \%$ to observe individual labeled chains separately. A mixed benzene solution of labeled and unlabeled PMMA at a total polymer concentration of $0.1 \mathrm{gl}^{-1}$ was spread on ultra pure water (NANO Pure II, Barnstead, Dubuque, IA, USA) at $20^{\circ} \mathrm{C}$ to form a monolayer on the water surface. The monolayer was compressed to a surface pressure of $5 \mathrm{mN} \mathrm{m}^{-1}$ and transferred onto a 40 $\mu \mathrm{m}$-thick film of unlabeled PMMA by the horizontal dipping method, which was prepared by casting from a 1,2-dichloroethane solution onto a clean glass plate. The sample film was annealed at $170{ }^{\circ} \mathrm{C}$ in vacuo. The structure of the sample film is illustrated in Figure 1.

\section{SNOM measurement}

Scanning near-field optical microscopy imaging was performed by a commercially available instrument ( $\alpha$-SNOM, WITec, Ulm, Germany) using a cantilever SNOM probe with a 60-nm aperture. A 438-nm laser beam (BCL-015440, CrystaLaser, Reno, NV, USA) was coupled into the sub-wavelength aperture to generate the optical near-field for the excitation of the perylene moiety in the PMMA-Pe chain. Fluorescence from the sample was collected by a microscope objective $(0.80 \mathrm{NA}, 60 \times$, Nikon, Tokyo, Japan) from the backside of the substrate and guided to a photomultiplier (H8631, Hamamatsu Photonics, Hamamatsu, Japan) through a long-pass filter (AELP 454, Omega Optical, Brattleboro, VT, USA). The SNOM probe was scanned with a contact mode on the sample surface, and the height image of the surface was simultaneously obtained using the fluorescence image. All SNOM measurements were carried out using the same probe in an ambient condition.

\section{RESULTS AND DISCUSSION}

Figure 2a shows the fluorescence SNOM image of a sample film before annealing. Individual PMMA-Pe chains embedded in the unlabeled PMMA monolayer were observed as bright spots in the fluorescence image. The height image of the sample surface for the same area showed no topographic features, indicating that the homogeneous deposition of the monolayer on the thick PMMA film and fluorescent

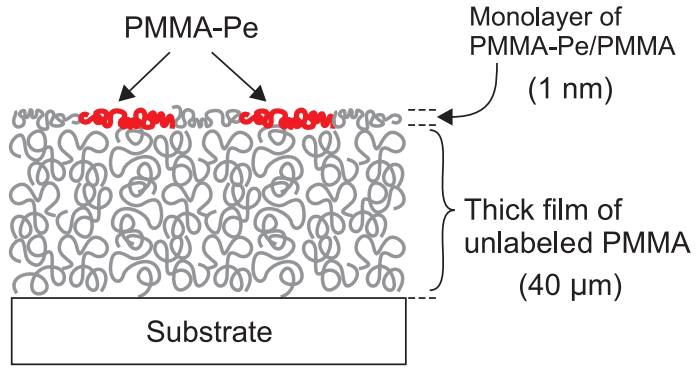

Figure 1 Schematic drawing of the structure of the poly(methyl methacrylate) monolayer deposited on a bulk film.

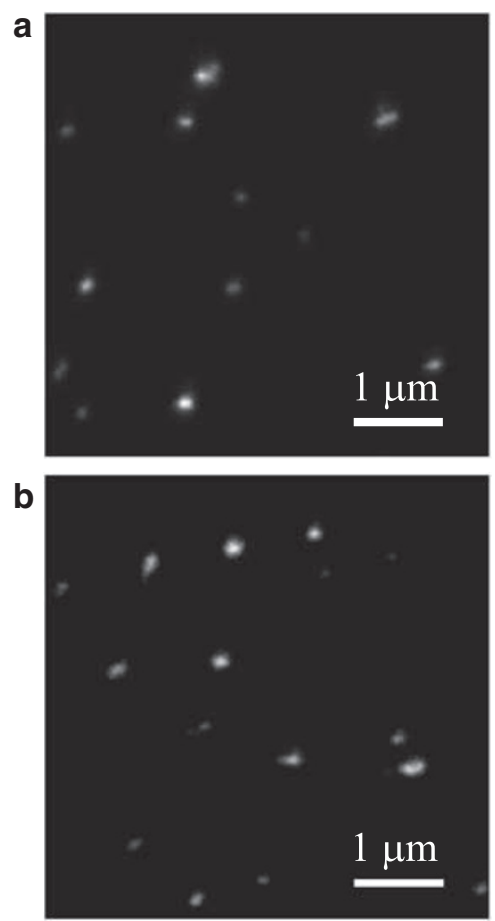

Figure 2 Fluorescence scanning near-field optical microscopy images of single poly(methyl methacrylate (PMMA) chains dispersed in the unlabeled PMMA matrix before (a) and after $48 \mathrm{~h}$ of annealing at $170^{\circ} \mathrm{C}(\mathrm{b})$

images are not influenced by surface topography (the topographic images are not shown here, but it is available as Supplementary information). Each fluorescence spot corresponds to a single PMMA-Pe chain, being confirmed from statistical analysis. ${ }^{21}$ The SNOM images in Figure 2 show a broad distribution of the fluorescence intensity from the single chain. This variation in signal intensity is due to the molecular weight distribution of the sample polymer. The number of dye moieties in a labeled chain is proportional to the length of the chain yielded by random copolymerization of methyl methacrylate and 3-perylenyl methyl methacrylate, ${ }^{21,24}$ resulting in the fluorescence intensity being proportional to molecular weight. Figure $2 \mathrm{~b}$ depicts the fluorescence SNOM image after annealing for $48 \mathrm{~h}$ at $170{ }^{\circ} \mathrm{C}$. In this large field image, the shapes and sizes of the spots after annealing are similar to those before annealing. To obtain more detailed information regarding chain conformation, the dimension of the single chain in the close-up image was analyzed. As the fluorescent moiety is randomly introduced along the PMMA-Pe chain as mentioned above, the fluorescence intensity at each pixel 
corresponds to the segment density therein. In the SNOM measurement, the optical near-field penetrates below the sample surface by a few hundred nanometers. Therefore, the chain conformation in the SNOM image is given as the projection of the individual chain onto the film plane. The squared radius of gyration for the projection of the PMMA chain in the two-dimensional plane, $R_{x y}{ }^{2}$, is evaluated by the second moment of the fluorescence image.

$$
\begin{aligned}
& R_{x y}^{2}=\frac{1}{I_{0}} \sum_{j} I_{j}\left(\mathbf{r}_{j}-\mathbf{r}_{0}\right)^{2}, \\
& I_{0}=\sum_{j} I_{j},
\end{aligned}
$$

where $\mathbf{r}_{j}$ and $I_{j}$ are the position vector and the fluorescence intensity for the $j$-th pixel in the SNOM image, and $\mathbf{r}_{0}$ is the position vector of the center of mass defined as

$$
\mathbf{r}_{0}=\frac{1}{I_{0}} \sum_{j} I_{j} \mathbf{r}_{j}
$$

$I_{0}$ corresponds to the total fluorescence intensity from a single chain. The kurtosis parameter, $K$, was also evaluated for each PMMA chain, ${ }^{30,31}$ which is related to the fourth moment of the fluorescence SNOM image (the equation is given in Supplementary information).

In this study, we discuss the annealing effect on chain conformation in terms of the parameters, $R_{x y}$ and $K$, evaluated from the SNOM image of individual chains. In the fluorescence SNOM measurements, the image is susceptible to photobleaching of the dye moiety. In the current experiment, SNOM imaging was carried out twice for the same area before and after annealing. If spatially inhomogeneous bleaching occurred in the single chain, the chain conformation would be distorted by repeated SNOM imaging. Therefore, before a detailed discussion on chain conformation, we examine the bleaching effect on the fluorescence image of the individual PMMA-Pe chains. Figure 3 shows the fluorescence SNOM images of a single PMMA-Pe chain, which are successively observed for the same chain. As they were acquired at room temperature (much lower than the glass transition temperature), a PMMA chain is unlikely to change its conformation in the measurement time of ca. $2 \mathrm{~h}$. The total fluorescence intensity $I_{0}$ from this chain decreased with the number of scannings: $2.0 \times 10^{5} \rightarrow$ $1.6 \times 10^{5} \rightarrow 1.4 \times 10^{5} \rightarrow 1.3 \times 10^{5}$, indicating that the dye moiety was bleached by the near-field illumination. On the other hand, the fluorescence image of the single chain seems to be the same after bleaching by several scannings. The values of $R_{x y}$ and $K$ were not affected by the decreased intensity at the single chain level. Table 1 summarizes the parameters of $R_{x y}$ and $K$ averaged over 50 chains, indicating the negligible effect of photobleaching on the conformation
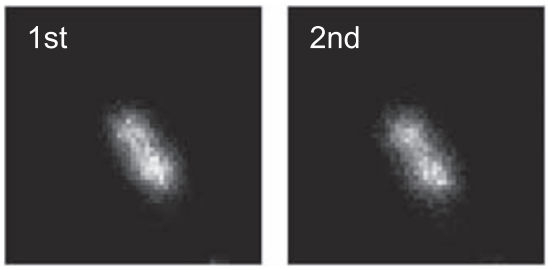

$$
\begin{aligned}
& I_{0}=2.0 \times 10^{5} \\
& R_{\text {xy }}=182 \mathrm{~nm} \\
& K=-1.18
\end{aligned}
$$

parameters. These quantities are normalized by signal intensity as shown in the definition (equation (1) and Supplementary equation $\mathrm{S} 1$ ). The dye molecules on the PMMA-Pe chain were homogeneously bleached in SNOM imaging; therefore, the chain conformation parameters, $R_{x y}$ and $K$, can be evaluated before and after annealing of the sample film.

Now we discuss the thermal effect on the conformation of the PMMA chain in a monolayer deposited on a bulk film. Figure 4 shows the histograms of $R_{x y}$ for 100 chains in the PMMA monolayer at annealing times of 0 and $48 \mathrm{~h}$. The histogram before annealing has a peak at $130 \mathrm{~nm}$ and a standard deviation of $28 \mathrm{~nm}$. After annealing for $48 \mathrm{~h}$, the distribution function of $R_{x y}$ showed a peak at $130 \mathrm{~nm}$, with a standard deviation of $30 \mathrm{~nm}$. Figure 5 summarizes the annealing time dependence of the average value of $R_{x y}$ clearly showing that the chain dimension in the lateral direction is not dependent on the annealing time. In terms of the ensemble-averaged $R_{x y}$ value, it seems that the chain conformation was not altered by annealing above the glass transition temperature.

For a more detailed discussion, we observed some polymer chains before and after annealing as depicted in Figure 6. On thermal activation, $R_{x y}$ for the PMMA chains varied from 89 to $87 \mathrm{~nm}$ for chain A in Figure 6, from 209 to $189 \mathrm{~nm}$ for B, and from 177 to $174 \mathrm{~nm}$ for C. The variation in $R_{x y}$ for most of the polymer chains was less than $10 \mathrm{~nm}$ and the largest change in $R_{x y}$ for the same single polymer chain before and after annealing was $20 \mathrm{~nm}$, which is smaller than the standard deviation of the conformational distribution. In terms of $R_{x y}$ the chain dimension in the lateral direction was not altered significantly. However, the spatial distribution of the segment density was different between chains before and after annealing. The brightness distribution before annealing for chains $\mathrm{B}$ and $\mathrm{C}$ looks homogeneous in the ellipsoidal area, but is enhanced at the center after annealing. To discuss the segment distribution for each polymer chain, we introduced the two-dimensional kurtosis, $K,^{30,31}$ which is related to the fourth moment of the intensity distribution. The kurtosis parameter is a measure of 'sharpness' relative to the Gaussian distribution; A higher value of $K$ indicates a narrower distribution, whereas a lower value of $K$ presents a broader distribution compared with a Gaussian function. After annealing for $48 \mathrm{~h}$,

\section{Table 1 Chain dimension parameters before and after annealing}

\begin{tabular}{lrcc}
\hline Scan number & $\mathrm{I} / 10^{4}$ & $\mathrm{R}_{\mathrm{xy}}(\mathrm{nm})$ & $\mathrm{K}$ \\
\hline 1 & 10.5 & 112 & -1.22 \\
2 & 7.5 & 113 & -1.20 \\
3 & 6.2 & 112 & -1.17 \\
4 & 5.5 & 112 & -1.20 \\
\hline
\end{tabular}

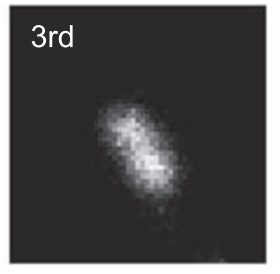

$$
\begin{aligned}
& I_{0}=1.4 \times 10^{5} \\
& R_{\text {xy }}=183 \mathrm{~nm} \\
& K=-1.17
\end{aligned}
$$

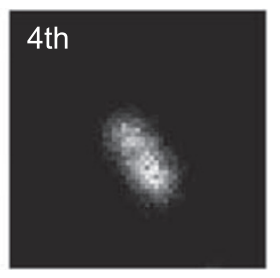

$I_{0}=1.3 \times 10^{5}$

$R_{\mathrm{xy}}=180 \mathrm{~nm}$

$K=-1.19$

Figure 3 Fluorescence scanning near-field optical microscopy images of a perylene-labeled poly(methyl methacrylate) chain sequentially scanned four times. Total fluorescence intensity $I_{0}$, chain dimension $R_{x y}$ and kurtosis $K$ evaluated from the fluorescence image are shown below each of them. 


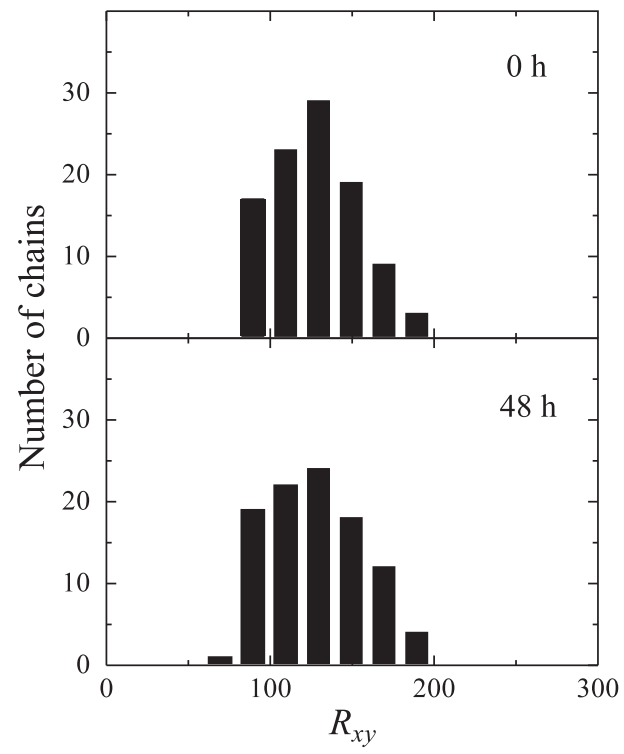

Figure 4 Histogram of the lateral chain dimension for poly(methyl methacrylate) chains before and after annealing.

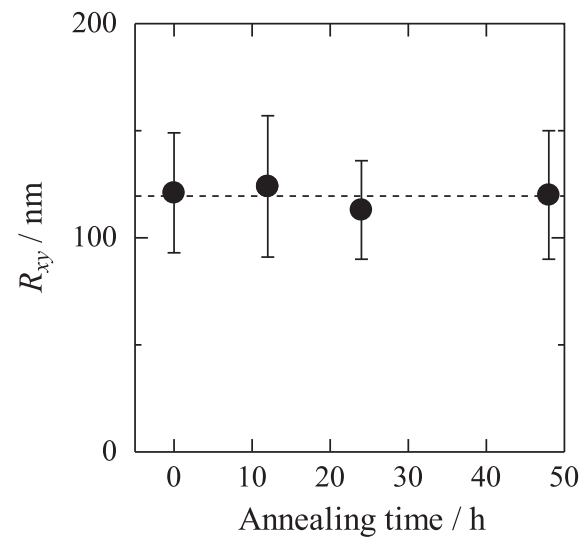

Figure 5 Annealing time dependence of the chain dimension of poly(methyl methacrylate) in a direction parallel to the film surface. The error bar indicates standard deviation.

the $K$ parameter increased from -1.29 to -1.22 for chain $\mathrm{A}$, from -1.77 to -1.28 for $\mathrm{B}$, and from -1.52 to -1.05 for $\mathrm{C}$. The increase in $K$ for the PMMA chains indicates that thermally induced conformational relaxation took place and the chain segment was rearranged to a Gaussian distribution around the center of the mass of the polymer chain. This result suggests that the two-dimensional conformation of the PMMA chain in a monolayer expands to the entropically stable three-dimensional random coil conformation, while keeping the chain dimension, $R_{x y}$ in the lateral direction.

To discuss the conformational change of the PMMA chains confined in a monolayer toward the bulk substrate, a cross-section of the specimen was observed by SNOM. The sample film was removed from the glass substrate and sliced into a thin specimen in a vertical direction to the surface plane with a microtome. The thickness of the specimen was $70 \mathrm{~nm}$. Figure 7 shows the fluorescence SNOM images and depth profiles of the fluorescence intensity. Just after deposition of the monolayer, the PMMA-Pe chain was localized at the sample surface as a single molecule monolayer with a thickness of $\sim 1 \mathrm{~nm} .{ }^{32}$ The SNOM image showed a much broader intensity profile compared with the actual thickness of the PMMA monolayer because of the spatial resolution of the apparatus. The SNOM image results from the convolution of the spatial distribution of the fluorescent object and the point spread function of the microscope. Because the thickness of the PMMA monolayer is negligible compared with the resolution of SNOM, the profile at $t=0$ corresponds to the point spread function of SNOM, and it was well fitted to a Gaussian profile, with the full width at half maximum of $100 \mathrm{~nm}$. On the other hand, the SNOM image after annealing showed an asymmetric profile, indicating that the PMMA-Pe segments on the surface diffused into the bulk substrate. Here, the fluorescence intensity profiles at various annealing times were analyzed. At first, we defined the $z$ axis in the depth direction toward the bulk substrate and set the origin at the position of the maximum fluorescence intensity. The distribution of the PMMA-Pe chain along the $z$ axis was modeled as,

$$
\rho(z)= \begin{cases}\rho_{0} \exp \left(-\frac{z^{2}}{2 \sigma^{2}}\right) & (z \geqslant 0) \\ 0 & (z<0)\end{cases}
$$

where $\sigma^{2}$ shows the spatial distribution of the chain segment of PMMA-Pe. Fluorescence intensity was fitted to the convolution of $\sigma(z)$ and point spread function to evaluate the best-fit value of $\sigma^{2}$. The broken line in Figure $7 \mathrm{~d}$ indicates the fitted profile. Figure 8 summarizes the annealing time dependence of $\sigma^{2}$, showing that $\sigma^{2}$ increases with time and then saturates at $48 \mathrm{~h}$. The polymer diffusion in bulk can be well described by the reptation model proposed by de Gennes $^{27}$ and Edwards. ${ }^{33}$ In this model, the polymer chain is constrained in a virtual tube composed of the entanglement network of neighboring chains, and the random motion occurs in the tube. The diffusion obeys Fick's law for a time that is longer than the reptation time $\tau$. In our system, the annealing time is far shorter than the reptation time, which was estimated to be $6 \times 10^{3} \mathrm{~h},{ }^{34}$ indicating that the observed motion is not characterized by reptational diffusion. Furthermore, the diffusion coefficient of the large molecular weight PMMA used in this experiment was estimated to be $10^{-17}$ to $10^{-18}$ at $170^{\circ} \mathrm{C},{ }^{27,34,35}$ whereas the apparent diffusion coefficient until $48 \mathrm{~h}$ in Figure 8 was $1 \times 10^{-16} \mathrm{~cm}^{2} \mathrm{~s}^{-1}$. The apparent diffusion coefficient is one or two orders larger than the translational diffusion coefficient of PMMA, indicating that we should consider a very fast diffusion mechanism besides the reptational one. Zhang and $\mathrm{Wool}^{36}$ examined the chain dynamics at the period shorter than the reptation time using both the Rouse and reptational relaxation models. They reported that the time range at $t<\tau$ involves the relaxation of nonequilibrium chain conformation and the segmental motion is dominant for the diffusion process. The polymer chain before annealing is in a nonequilibrium state because the chain is constrained on the surface and takes a twodimensional conformation. Hence, the increase in $\sigma^{2}$ before $48 \mathrm{~h}$ in Figure 8 shows conformational relaxation from a two-dimensional conformation to a more entropically favorable three-dimensional conformation by thermal activation.

Figures $9 \mathrm{a}$ and $\mathrm{b}$ illustrate the conformation of the polymer chain confined in a monolayer and that during the rearrangement process from a two-dimensional form to a three-dimensional one, respectively. The polymer chain in a monolayer is not allowed to have a crossover with other chains because of constraint in the quasi-two-dimensional plane. Therefore, the polymer chain tends to exclude others in order not to make the free space in plane, and consequently it takes a selfcontracted conformation. Such a contracted conformation of the twodimensional polymer chain has been predicted by de Gennes' scaling theory ${ }^{37}$ and has been reported by several experiments so far. ${ }^{38,39}$ Although each chain is segregated from others in plane, it has to 

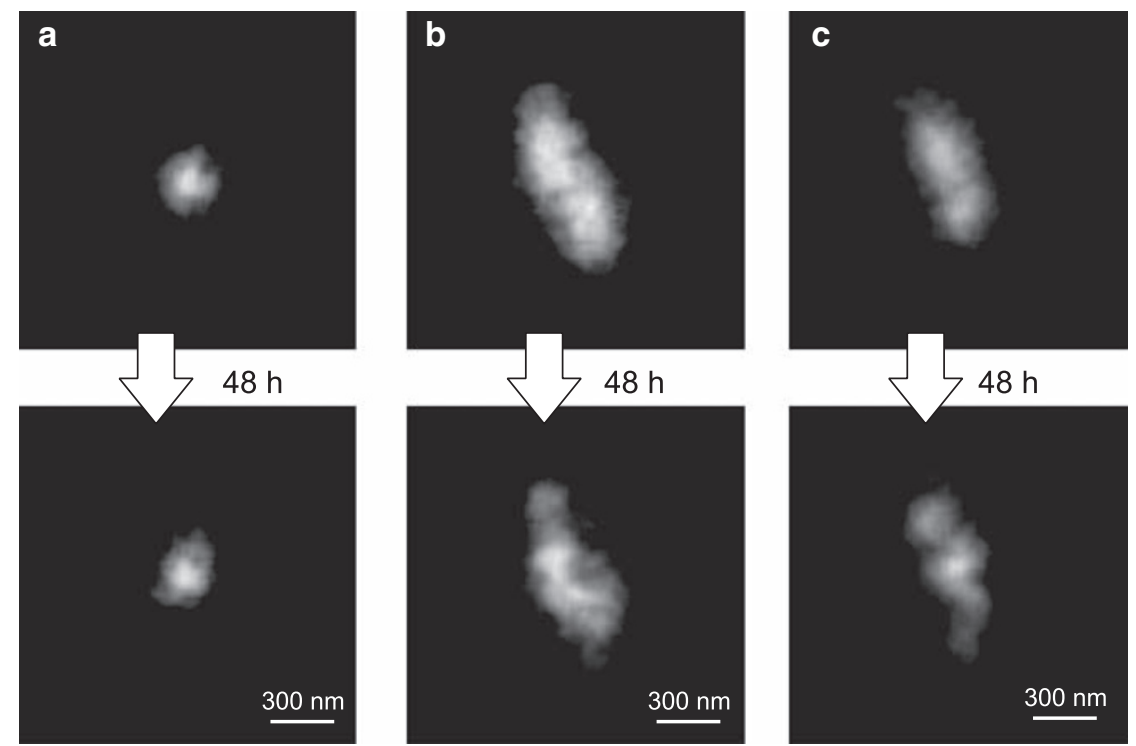

Figure 6 Fluorescence scanning near-field optical microscopy images of the same single poly(methyl methacrylate) chains before and after annealing for $48 \mathrm{~h}$ (the top and bottom sides, respectively). Chain A: $R_{x y}=89 \mathrm{~nm}, K=-1.29$ (top) and $R_{x y}=87 \mathrm{~nm} ; K=-1.22$ (bottom). Chain B: $R_{x y}=209 \mathrm{~nm} ; K=-1.77$ (top) and $R_{x y}=189 \mathrm{~nm} ; K=-1.28$ (bottom). Chain C: $R_{x y}=177 \mathrm{~nm} ; K=-1.52$ (top) and $R_{x} y=174 \mathrm{~nm} ; K=-1.05$ (bottom). The dimension of all images is $1.5 \times 1.5 \mu \mathrm{m}$.

a
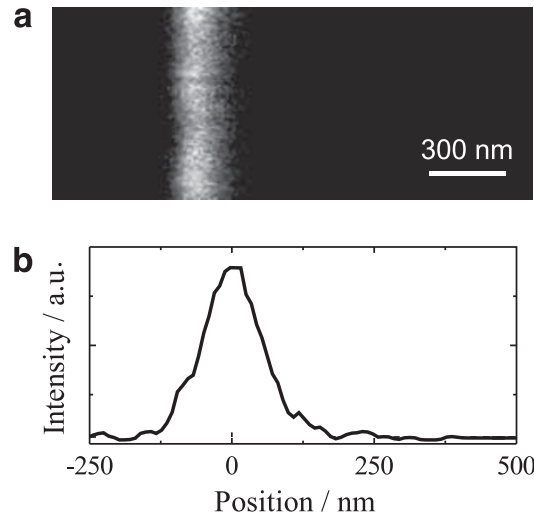

C
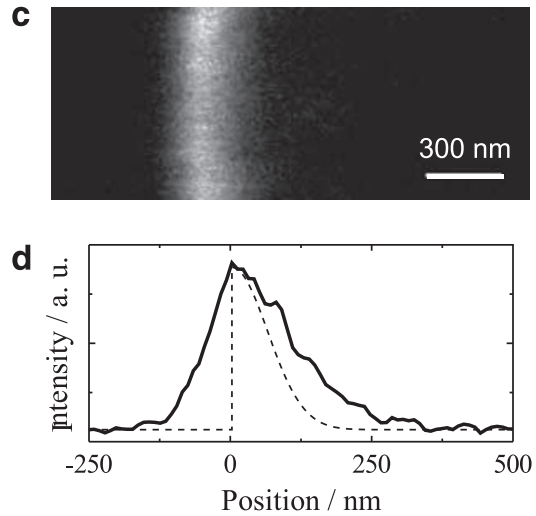

Figure 7 Scanning near-field optical microscopy images observed from the normal direction to the sample surface before $(\mathbf{a}, \mathbf{b})$ and after annealing for $48 \mathrm{~h}$ (c, d): fluorescence images (a, c) and cross-section profiles of the fluorescence image (b, d). The solid and broken lines in panels $b$ and $d$ indicate the experimentally obtained fluorescence intensity and the profiles deconvoluted with point spread function, respectively.

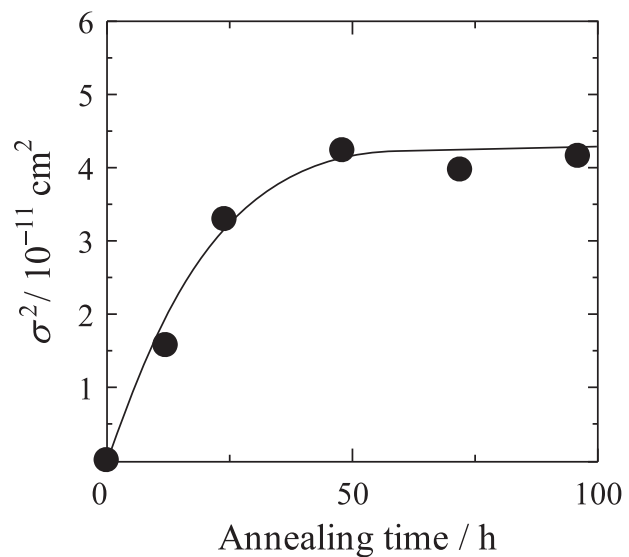

Figure 8 Annealing time dependence of the spatial distribution of the chain segment of perylene-labeled poly(methyl methacrylate).

cover a relatively large area of the surface as determined by the limiting molecular area of the monomer unit and the degree of polymerization, because the volume of the chain and the density of the ultrathin film must be constant even in low dimensions. Considering these two-dimensional characteristics mentioned above, it can be safely said that polymer segments are homogeneously distributed inside a single polymer domain with a relatively large area. However, once the polymer chain is free from restriction on the bulk substrate of the same polymer, it starts interlacing with other chains and relaxes into a three-dimensional random coil. The mixing with surrounding polymer chains causes chain expansion mainly to the depth direction, which is the dimension newly released by deposition. Although our experimental time range is still too short to attain the equilibrium state, the present results clearly show the characteristics of the two-dimensional polymer chains and the conformational rearrangement process to an entropically favorable form in three dimensions. 
a Before annealing

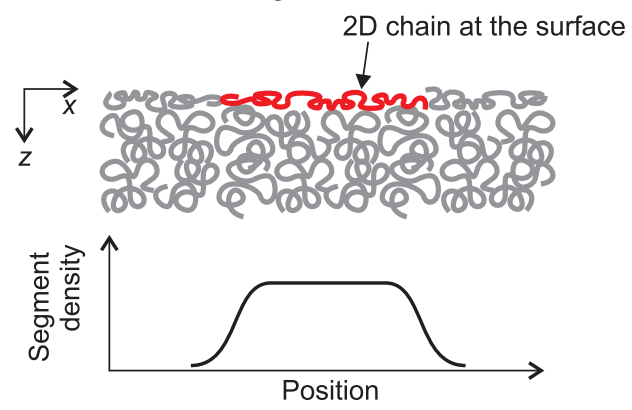

b After annealing
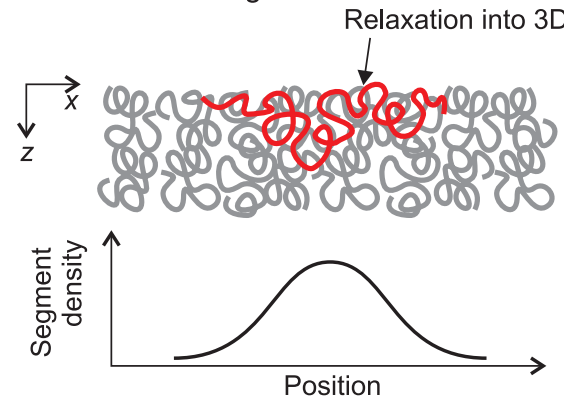

Figure 9 Schematic illustration of thermally induced conformational relaxation from a two-dimensional form (a) to a three-dimensional one (b). Probe chains are shown as red contours in the upper parts in each panel. The lower sides of panels $a$ and $b$ show schematic profiles of the segment density of single polymer chains before and after annealing, respectively.

\section{CONCLUSIONS}

Real SNOM images of individual PMMA chains allowed us to discuss the characteristics of two-dimensional polymer chains and their conformational rearrangement after deposition on a solid substrate. The results obtained from this study are summarized as follows: (1) conformation of polymer chains during rearrangement from a twodimensional monolayer to three-dimensional random coils was directly observed by SNOM. (2) The averaged chain dimension in the lateral direction was not significantly altered by thermal treatment. (3) The chain segment was rearranged to a Gaussian distribution around the center of the mass of the polymer chain after annealing. (4) Polymer chains penetrated into the bulk substrate at a rate faster than that of the translational diffusion of the entire polymer chains.

There are still intensive arguments on the particular characteristics of polymeric materials at the surface/interface and ultrathin films thinner than the molecular dimensions, because it is critical for the design and fabrication of nanometric architectures. This study revealed the relaxation mechanism of low-dimensional chains in such a restricted space, and also the significance of direct imaging at the molecular level for investigating these advanced research fields.

\section{ACKNOWLEDGEMENTS}

This work was supported by Grant-in-Aids from the Japan Society for the Promotion of Science (JSPS) and by the Ministry of Education, Culture, Sports, Science and Technology (MEXT), Japan. We also thank the Global COE Program, 'International Center for Integrated Research and Advanced Education in Material Science,' and the Innovative Techno-Hub for Integrated Medical Bio-imaging Project of the Special Coordination Funds for Promoting Science and Technology from MEXT.
1 Tredgold, R. H. Langmuir-Blodgett films made from preformed polymers. Thin Solid Films 152, 223-230 (1987).

2 Ito, S., Mabuchi, M., Sato, N. \& Aoki, H. Development of ultrathin polymer films and their characteristics in two dimensions. Bull. Chem. Soc. Jpn. 78, 371-382 (2005).

3 Schneider, J., Ringsdorf, H. \& Rabolt, J. F. Structural studies of polymers with hydrophilic spacer groups: infrared spectroscopy of Langmuir-Blodgett multilayers of preformed polymers with hydrocarbon side chains. Macromolecules 22, 205-210 (1989).

4 Schneider, J., Erdelen, C., Ringsdorf, H. \& Rabolt, J. F. Structural studies of polymers with hydrophilic spacer groups. 2. Infrared spectroscopy of Langmuir-Blodgett multilayers of polymers with fluorocarbon side chains at ambient and elevated temperatures. Macromolecules 22, 3475-3480 (1989).

5 Hsiung, H., Beckerbauer, R. \& Rodriguez Parada, J. M. Orientational disordering transition in Langmuir-Blodgett films of fluorinated polymers. Langmuir 9, 1971-1973 (1993).

6 Ringsdorf, H., Schmidt, G. \& Schneider, J. Oriented ultrathin membranes from monomeric and polymeric amphiphiles: monolayers, liposomes and multilayers. Thin Solid Films 152, 207-222 (1987).

7 Biddle, M. B., Lando, J. B., Ringsdorf, H., Schmidt, G. \& Schneider, J. Polymeric amphiphiles with hydrophilic main chain spacers: studies in monolayers and LangmuirBlodgett multilayers. Colloid Polym. Sci. 266, 806-813 (1988).

8 See, Y. K., Cha, J., Chang, T. \& Ree, M. Glass transition temperature of Poly(tert-butyl methacrylate) Langmuir? Blodgett film and spin-coated film by X-ray reflectivity and ellipsometry. Langmuir 16, 2351-2355 (2000).

9 Kügler, R. \& Knoll, W. Influence of the polymethacrylate chain conformation in Langmuir-Blodgett-Kuhn films on the glass-transition temperature. Macromol. Chem. Phys. 203, 923-930 (2002).

10 Mabuchi, M., Kawano, K., Ito, S., Yamamoto, M., Takahashi, M. \& Masuda, T. Structural relaxation of ultrathin polymer films prepared by the Langmuir-Blodgett technique: characteristics of the two-dimensional system. Macromolecules 31, 60836088 (1998).

11 Mabuchi, M., Kawano, K., Ito, S. \& Yamamoto, M. Structural relaxation of LangmuirBlodgett films of Poly(vinyl octanal acetal) with various tacticities. Macromolecules 31, 6077-6082 (1998).

12 Kumaki, J., Nishikawa, Y. \& Hashimoto, T. Visualization of single-chain conformations of a synthetic polymer with atomic force microscopy. J. Am. Chem. Soc. 118 , 3321-3322 (1996).

13 Kumaki, J. \& Hashimoto, T. Conformational change in an isolated single synthetic polymer chain on a mica surface observed by atomic force microscopy. J. Am. Chem. Soc. 125, 4907-4917 (2003).

14 Roiter, Y. \& Minko, S. AFM single molecule experiments at the solid-liquid interface: in situ conformation of adsorbed flexible polyelectrolyte chains. J. Am. Chem. Soc. 127, 15688-15689 (2005).

15 Maier, B. \& Rädler, J. O. Conformation and self-diffusion of single DNA molecules confined to two dimensions. Phys. Rev. Lett. 82, 1911-1914 (1999).

16 Maier, B. \& Rädler, J.O. Shape of self-avoiding walks in two dimensions. Macromolecules 34, 5723-5724 (2001).

17 Abbe, E. Beitrage zur Theorie des Mikroskops und der Mikroskopischen Wahrnehmung. Arch. Mikrosk. Anat. 9, 413-468 (1873).

18 Betzig, E. \& Trautman, J. K. Near-field optics: microscopy, spectroscopy, and surface modification beyond the diffraction limit. Science 257, 189-195 (1992).

19 Ohtsu, M. Near-field Nano/Atom Optics and Technology (Springer, Tokyo, 1998).

20 Paesler, M. A. \& Moyer, P. J. Near-Field Optics: Theory, Instrumentation, and Applications (Wiley, New York, 1996).

21 Aoki, H., Anryu, M. \& Ito, S. Two-dimensional polymers investigated by scanning nearfield optical microscopy: conformation of single polymer chain in monolayer. Polymer 46, 5896-5902 (2005).

22 Ube, T., Aoki, H., Ito, S., Horinaka, J. \& Takigawa, T. Conformation of single PMMA chain in uniaxially stretched film studied by scanning near-field optical microscopy. Polymer 48, 6221-6225 (2007).

23 Yang, J., Sekine, R., Aoki, H. \& Ito, S. Localization and orientation of homopolymer in block copolymer lamella: a near-field optical microscopy study. Macromolecules 40, 7573-7580 (2007).

24 Aoki, H., Morita, S., Sekine, R. \& Ito, S. Conformation of single Poly(methyl methacrylate) chains in an ultra-thin film studied by scanning near-field optical microscopy. Polym. J. 40, 274-280 (2008).

25 Sekine, R., Aoki, H. \& Ito, S. Conformation of single block copolymer chain in twodimensional microphase-separated structure studied by scanning near-field optical microscopy. J. Phys. Chem. B 113, 7095-7100 (2009).

26 Tamai, Y., Sekine, R., Aoki, H. \& Ito, S. Conformation of single homopolymer chain in microphase-separated block copolymer monolayer studied by scanning near-field optical microscopy. Macromolecules 42, 4224-4229 (2009).

27 de Gennes, P. G. Reptation of a polymer chain in the presence of fixed obstacles. J. Chem. Phys. 55, 572-579 (1971).

28 Brinkhuis, R. H. G. \& Schouten, A.J. Thin-film behavior of Poly(methyl methacrylates). 1. Monolayers at the air-water interface. Macromolecules 24, 1487-1495 (1991).

29 Aoki, H. \& Ito, S. Two-dimensional polymers investigated by scanning near-field optical microscopy: phase separation of polymer blend monolayer. J. Phys. Chem. B 105, 4558-4564 (2001).

30 Zhang, N. F., Postek, M. T., Larrabee, R. D., Vladár, A. E., Keery, W. J. \& Jones, S. N. Image sharpness measurement in the scanning electron microscope-Part III. Scanning 21, 246-252 (1999). 
31 Mardia, K. V. Measures of multivariate Skewness and Kurtosis with applications. Biometrika 57, 519-530 (1970).

32 Gabrielli, G., Puggelli, M. \& Baglioni, P. Orientation and compatibility in monolayers: II. Mixtures of polymers. J. Colloid Interf. Sci. 86, 485-500 (1982).

33 Edwards, S. F. The statistical mechanics of polymerized material. Proc. Phys. Soc. 92 , 9-16 (1967).

34 Masuda, T., Kitagawa, K. \& Onogi, S. Viscoelastic properties of Poly(methyl methacrylates) prepared by anionic polymerization. Polym. J. 1, 418-424 (1970).

35 Liu, Y., Reiter, G., Kunz, K. \& Stamm, M. Investigation of the interdiffusion between Poly(methyl methacrylate) films by marker movement. Macromolecules 26, 2134 2136 (1993).
36 Zhang, H. \& Wool, R. P. Concentration profile for a polymer-polymer interface. 1. Identical chemical composition and molecular weight. Macromolecules 22, 30183021 (1989).

37 de Gennes, P. G. Scaling Concepts in Polymer Physics (Cornell University Press, New York, 1979).

38 Sato, N., Ito, S. \& Yamamoto, M. Interfacial behavior of acetalized Poly(vinyl alcohol) monolayers observed by brewster angle microscopy and canal surface viscometry. Polym. J. 28, 784-789 (1996).

39 Sato, N., Ito, S. \& Yamamoto, M. Molecular weight dependence of shear viscosity of a polymer monolayer: evidence for the lack of chain entanglement in the two-dimensional plane. Macromolecules 31, 2673-2675 (1998).

Supplementary Information accompanies the paper on Polymer Journal website (http://www.nature.com/pj) 